Rev. Biol. Trop., 48(2/3): 569-578, 2000

www.ucr.ac.cr www.ots.ac.cr www.ots.duke.edu

\title{
Anatomía y usos de la madera de siete árboles tropicales de México
}

Silvia Rebollar y Alejandra Quintanar

Departamento de Biología, Universidad Autónoma Metropolitana-Iztapalapa. A.P.55-535. 09340 México, D. F. Fax: 58-04-46-88. Correo electrónico sired@xanum.uam.mx

Recibido 30-VI-1999. Corregido 25-I-1999. Aceptado 20-I-2000.

\begin{abstract}
The wood anatomy of Coccoloba cozumelensis Hemsl., Coccoloba spicata Lundell, Gymnanthes lucida Sw., Blomia cupanioides Miranda, Canella winterana (L.) Gaertn., Aspidosperma megalocarpon Müell Arg. and Ehretia tinifolia L., is described. One tree per species was collected in the tropical rain forest of Quintana Roo, Mexico. Their wood has important traditional uses in furniture, tools, rural buildings, posts, fences, railroads and firewood. Macroscopic and microscopic characteristics were described and measured in wood samples, permanent slides and macerated material. These species have diffuse porosity, alternate vessel pits, simple perforation plates, numerous and small rays; libriform fibres are common, as well as ergastic material in gum forms, calcium carbonate and silica crystals. These characteristics explain aesthetical, weigth, hardness and resistance (to mechanical and biological damage) characters that fit traditional use by the Maya.
\end{abstract}

Kew words: Wood anatomy, tropical rain forest, uses, Maya, México.

Los árboles de las especies seleccionadas para este estudio, están representados en la selva mediana subperennifolia de tres municipios del estado de Quintana Roo, México. Han sido utilizados tradicionalmente por los campesinos de las localidades de recolección en diversas formas, particularmente E. tinifolia como leña, (Terán y Rasmussen 1994), B. cupanioides en la fabricación de muebles (especialmente sillas), A. megalocarpon para durmientes, G. lucida en postes de cercas (Escalante 1986) y desde tiempos prehispánicos en la construcción rural de casas habitación, como es el caso de C. cozumelensis y C.spicata (Villers et al. 1981, Escalante 1986). La madera de estas especies es de atractivos colores y veteados, las características anatómicas distintivas para las especies son: su porosidad difusa, los poros numerosos y pequeños, rayos numerosos y bajos, fibras del tipo libriforme y la presencia de extractivos; estos caracteres les confieren a las especies ser altamente decorativas, por su peso y dureza son resistentes a la pudrición y muy durables por lo que pueden ser consideradas para usos diversos. En México, se ha estudiado la anatomía de la madera de: Aspidosperma megalocarpon procedente de Ocosingo, Chiapas por De la Paz Pérez y Corral (1980), la de Coccoloba barbadensis por De la Paz Pérez et al. (1980) y la de Gymnanthes lucida por Rogel (1982) ambas de San Felipe Bacalar, Quintana Roo; también se conocen las características estéticas y usos artesanales de Ehretia. latifolia, procedente de Pátzcuaro, Michoacán, estudiada por Guridi (1980).

\section{MATERIALES Y MÉTODOS}

La madera de las especies estudiadas se obtuvo de árboles seleccionados, sanos, maduros, de fustes rectos, uno para cada especie, 
con alturas de $13-15 \mathrm{~m}$ y diámetros de $22-50$ $\mathrm{cm}$, los que se colectaron de acuerdo a las especificaciones de Ramos y Díaz (1981) en la selva mediana subperennifolia de tres municipios de Quintana Roo: en Puerto Morelos, municipio de Benito Juárez a $C$. winterana, $C$. cozumelensis y G. lucida; en el ejido de Noh-Bec municipio de Felipe Carrillo Puerto a $B$. cupanioides y en el ejido de Tres Garantías, municipio de Othón P. Blanco, A. megalocarpon, $C$. spicata y E. Tinifolia.

El estudio anatómico microscópico se realizó en preparaciones fijas de los tres cortes típicos de la madera (transversal, tangencial y radial) y en material disociado, el macroscópico en tablillas de xiloteca de $15 \times 7$ $X 1 \mathrm{~cm}$; la metodología en detalle se da en Rebollar et al. (1987, 1993). La denominación de las características macroscópicas se hizo de acuerdo con Tortorelli (1956) y para el color se usaron las tablas de Munsell (Anónimo 1954). La nomenclatura utilizada en las descripciones microscópicas es la de IAWA Committee (Anónimo 1989) y para los rayos se usó la de Kribs (1968).

A los elementos mensurables se les hizo un análisis estadístico univariado con un error de muestreo del $5 \%$ y se denominaron con base en la media de acuerdo a la clasificación de Chattaway (1932) y la de IAWA Committee (Anónimo 1937, 1939). Para el conteo del número de poros y de rayos se usan de 10 a 15 campos con el objetivo de $10 \mathrm{X}$; el valor para el número de poros se da en $\left(/ \mathrm{mm}^{2}\right)$, para el número de rayos en milímetros lineales $(/ \mathrm{mm})$ y para el resto de los caracteres en micrones $(\mu \mathrm{m})$.

Las descripciones anatómicas de las especies se presentan en orden filogenético según la clasificación de Engler modificada por De Dalla Torre \& Harms (1963). Los usos se obtuvieron por fuentes bibliográficas: Andrade (1976), De la Paz Pérez y Corral (1980) y De la Paz Pérez et al. (1980), Guridi (1980), Echenique-Manrique (1970), Herrera et al. (1982), Huerta y Becerra (1982), Rogel (1982); los tradicionales por comunicación verbal de campesinos mayas de cada localidad de colecta y los sugeridos de acuerdo a sus características anatómicas.

\section{RESULTADOS}

\section{Coccoloba cozumelensis}

Familia: Polygonaceae

Nombres comunes: Ch' iich' boob, kaanbal boob, sak boob (Sosa et al. 1985).

Distribución en México: Península de Yucatán y Chiapas (Standley \& Steyermark 1946).

Características anatómicas de la madera:

a. Estéticas. La madera presenta diferencia de color entre albura y duramen, la albura es rosa (5YR 7/3) y el duramen castaño rojizo oscuro (5YR 3/3), no tiene olor ni sabor característicos; su brillo es mediano, de veteado pronunciado, textura mediana e hilo entrecruzado. Las zonas de crecimiento están marcadas por fibras.

b. Macroscópicas y microscópicas (Figs. 1. a-c). Todos los elementos constitutivos son visibles con lupa $(6 \mathrm{X})$. Los poros son de distribución difusa, la mayoría múltiples radiales de 2 a 5 y algunos solitarios, numerosos de 14 (11-16)/mm2 y de diámetro tangencial moderadamente pequeño de 88 (79-95) $\mu \mathrm{m}$.

Los elementos de vaso son medianos de 380 (345-410) $\mu \mathrm{m}$, sus paredes muestran puntuaciones areoladas alternas y opuestas y placa perforada simple. Algunos presentan gomas.

El parénquima es difuso y paratraqueal escaso. La mayoría de las células con cristales romboidales.

Los rayos son uniseriados 1 (1-3), heterogéneos, muy numerosos de $12(10-14) / \mathrm{mm}$, extremadamente bajos de 295 (250-315) $\mu \mathrm{m}$, muy finos de 16 (13-19) $\mu \mathrm{m}$. La mayoría de las células presenta gomas.

Las fibras son de tipo libriforme, moderadamente cortas de 830 (795-897) $\mu \mathrm{m}$, de diámetro fino de 20 (17-21) $\mu \mathrm{m}$ y pared delgada de $6(6-8) \mu \mathrm{m}$. Algunas presentan gomas.

\section{Coccoloba spicata}

Familia: Polygonaceae

Nombres comunes: boob, boobchi che', xboob che' (Sosa et al. 1985). 

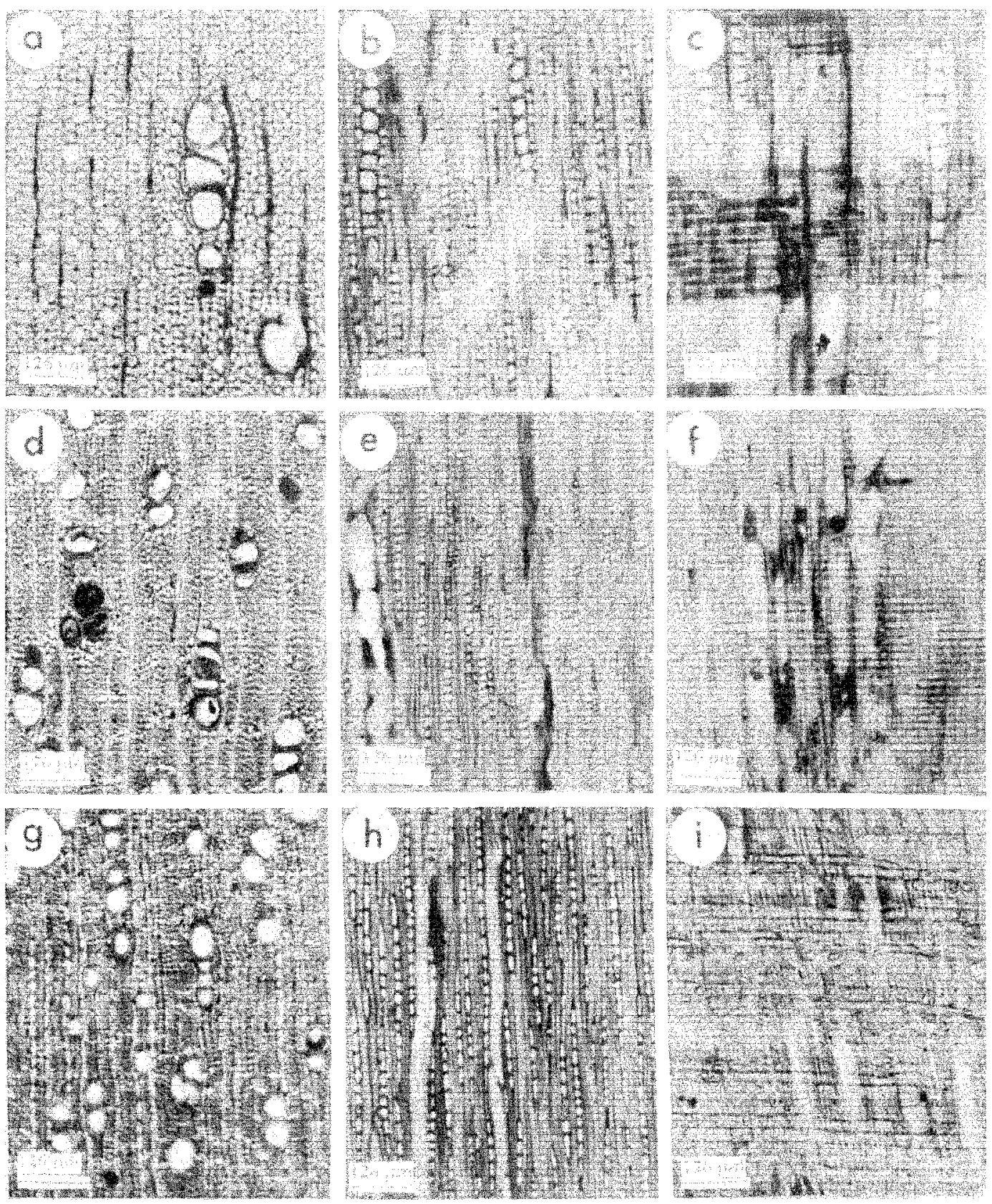

Fig. 1. Corte transversal (izquierda), corte tangencial (centro), corte radial (derecha). Figs. a-c. Coccoloba cozumelensis. Figs. d-f. Coccoloba spicata. Figs. g-i. Gymnanthes lucida.

Fig. 1. Transverse section (left), tangential section (centre), radial section (right). Figs. a-c Coccoloba cozumelensis. Figs. d-f. Coccoloba spicata. Figs. g-i Gymnanthes lucida. 
Distribución en México: Yucatán, Quintana Roo (Standley \& Steyermark 1946a), (Sosa et al. 1985).

Características anatómicas de la madera:

a. Estéticas. La madera no presenta diferencia de color entre albura y duramen, es de tonalidades gris rojizo (5YR 5/2) y castaño pálido (10YR6/3), no tiene olor ni sabor característicos, su brillo es mediano, de veteado suave, textura fina e hilo entrecruzado. Las zonas de crecimiento están marcadas por parénquima axial.

b. Macroscópicas y microscópicas. (Figs. 1.d-f). Todos los elementos constitutivos son visibles con lupa (6X). Los poros son de distribución difusa, la mayoría múltiples radiales de 2 a 5 y solitarios, numerosos de 36 (28$41) / \mathrm{mm}^{2}$ y de diámetro tangencial moderadamente pequeño de 72 (68-76) $\mu \mathrm{m}$. Los elementos de vaso son medianos de 480 (436-512) $\mu \mathrm{m}$, sus paredes muestran puntuaciones areoladas alternas y opuestas y placa perforada simple. La mayoría presenta gomas.

El parénquima es paratraqueal en bandas angostas. La mayoría de las células presentan cristales romboidales.

Los rayos son uniseriados, la mayoría homogéneos y algunos rayos con células cuadradas, muy numerosos de 14 (13-16)/mm, extremadamente bajos de 240 (235-265) $\mu \mathrm{m}$ y muy finos de 24 (22-28) $\mu \mathrm{m}$. Algunos presentan gomas.

Las fibras son de tipo libriforme, extremadamente cortas de 430 (413-441) $\mu \mathrm{m}$, de diámetro fino de 13 (10-15) $\mu \mathrm{m}$, y pared delgada de 4 (2-4) $\mu \mathrm{m}$. Algunas presentan gomas.

\section{Gymnanthes lucida}

Familia: Euphorbiaceae

Nombrescomunes: yaitil (Sosa et al. 1985).

Distribución en México: Península de Yucatán (Standley \& Steyermark 1949).

Características anatómicas de la madera:

a. Estéticas. La madera presenta diferencia de color entre albura y duramen, la albura es amarilla $(2.5 \mathrm{Y} 8 / 6)$ y el duramen es castaño amarillo (10 YR 5/4) no tiene olor ni sabor característicos, su brillo es mediano, de vetea- do pronunciado, textura fina e hilo entrecruzado. Las zonas de crecimiento están marcadas por fibras.

b. Macroscópicas y microscópicas. (Figs.1. g-i). Todos los elementos constitutivos son visibles con lupa (6 X). Los poros son de distribución difusa, múltiples radiales de 2 a 12 $\mathrm{y}$ algunos solitarios, muy numerosos de 42 (38-44)/ $\mathrm{mm}^{2}$ y de diámetro tangencial moderadamente pequeño de 60 (53-72) $\mu \mathrm{m}$. Los elementos de vaso son medianos de 400 (380430) $\mu \mathrm{m}$, sus paredes muestran puntuaciones areoladas alternas y placa perforada simple. Algunos presentan gomas.

El parénquima es difuso en agregados, formado por hileras de 4-5 células.

Los rayos son uniseriados, heterogéneos, formados alternadamente por células cuadradas y procumbentes, muy numerosos de 20 $(18-22) / \mathrm{mm}$, extremadamente bajos de 400 (370-423) $\mu \mathrm{m}$ y muy finos de $16(13-18) \mu \mathrm{m}$. La mayoría presenta cristales romboidales y amorfos.

Las fibras son de tipo libriforme, moderadamente cortas de $420(412-435) \mu \mathrm{m}$, de diámetro fino de 16 (14-18) $\mu \mathrm{m}$ y pared gruesa de 8 (6-9) $\mu \mathrm{m}$. Algunas presentan gomas.

\section{Blomia cupanioides}

Familia: Sapindaceae

Nombres comunes: palo de sol

Distribución en México: Ocosingo, Chiapas, (Miranda 1953). Chiapas y Tabasco (Martínez 1979).

Características anatómicas de la madera:

a. Estéticas. La madera no presenta diferencia de color entre la albura y el duramen, es de color castaño muy pálido (7.5 YR 8/6), no tiene olor ni sabor característicos, su brillo es bajo, de veteado suave, textura fina e hilo recto. Las zonas de crecimiento no están marcadas.

b. Macroscópicas y microscópicas. (Figs. 2. a-c). Todos los elementos constitutivos son visibles con lupa $(6 \mathrm{X})$. Los poros son de distribución difusa, exclusivamente solitarios, numerosos de $32(27-43) / \mathrm{mm}^{2}$ y de diámetro tangencial muy pequeño de 40 (35-43) $\mu \mathrm{m}$. 

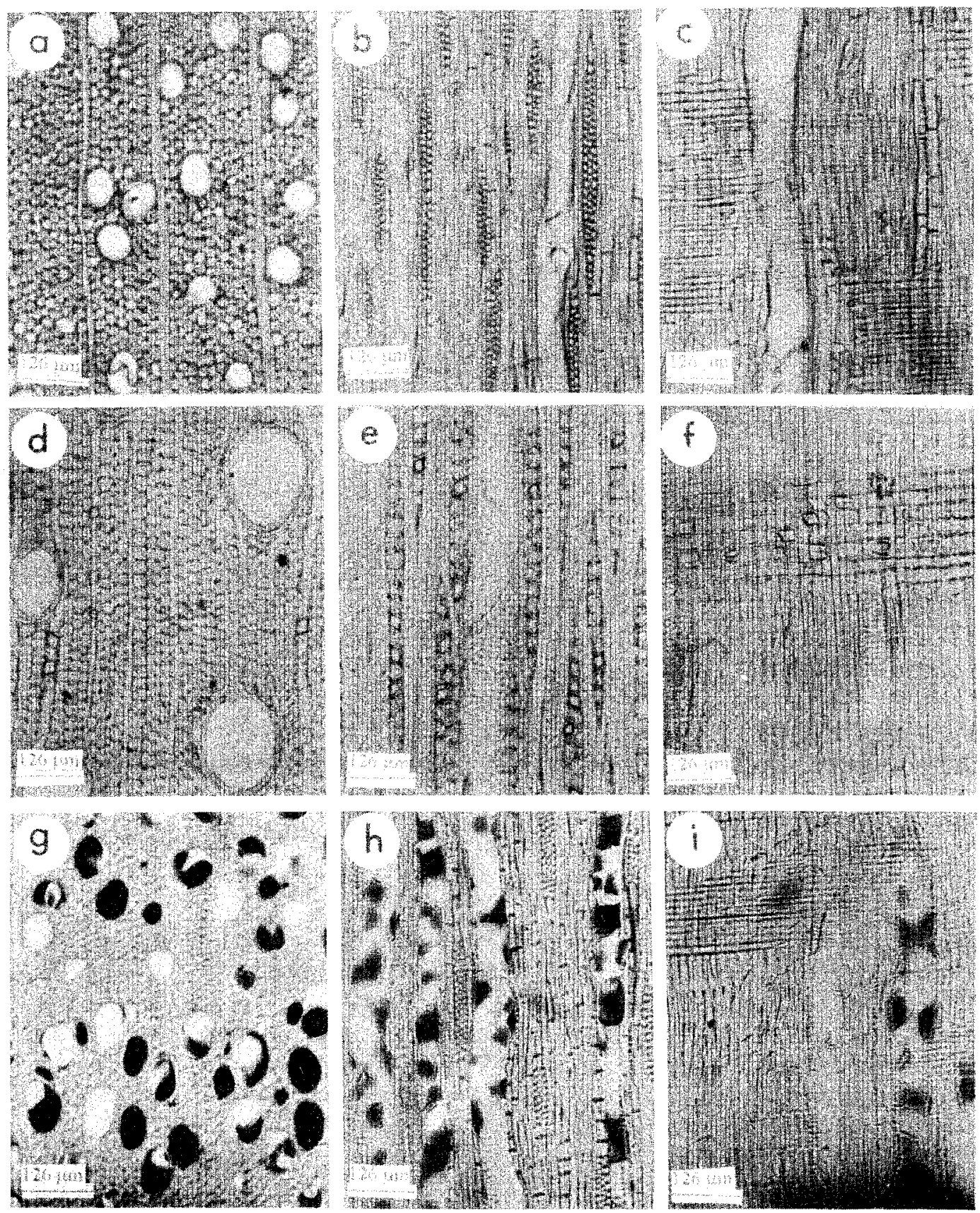

Fig. 2. Corte transversal (izquierda), corte tangencial (centro), corte radial (derecha). Figs. a-c Blomia cupanioides. Figs. d-f. Canella winterana. Figs. g-i. Aspidosperma megalocarpon.

Fig. 2. Transverse section (left), tangential section (centre), radial section (right). Figs. a-c. Blomia cupanioides. Figs. d-f. Canella winterana. Figs. g-i. Aspidosperma megalocarpon. 
Los elementos de vaso son extremadamente cortos de 68 (65-75) $\mu \mathrm{m}$, sus paredes muestran puntuaciones areoladas alternas $\mathrm{y}$ placa perforada simple.

El parénquima es difuso en agregados y paratraqueal escaso. La mayoría presenta cristales romboidales.

Los rayos son biseriados 2 (1-2), heterogéneos, moderadamente numerosos de $6(5-8) /$ mm, extremadamente bajos de 420 (415-426) $\mu \mathrm{m}$ y moderadamente finos de 43 (38-46) $\mu \mathrm{m}$.

Las fibras son de tipo libriforme, extremadamente cortas de 362 (356-375) $\mu \mathrm{m}$, de diámetro mediano de 30 (27-33) $\mu \mathrm{m}$ y pared muy delgada de 8 (5-9) $\mu \mathrm{m}$. Algunas presentan gomas.

\section{Canella winterana}

Familia: Canellaceae

Nombres comunes: canelo y cuyo.

Distribución en México: Quintana Roo, Indias Occidentales (Index Kewensis 1997).

Características anatómicas de la madera:

a. Estéticas. La madera presenta diferencia de color entre albura y duramen, la albura es castaño amarillo claro (2.5 YR 6/4) y el duramen presenta tonalidades negras $(5 Y 2.5 / 2)$ y castaño olivo claro (2.5Y 5/4), no tiene olor ni sabor característicos, su brillo es bajo, de veteado pronunciado, textura mediana e hilo entrecruzado. Las zonas de crecimiento están marcadas por parénquima marginal y fibras.

b. Macroscópicas y microscópicas. (Figs. 2. d-f). Todos los elementos constitutivos son visibles con lupa (6X). Los poros son de distribución difusa, la mayoría múltiples radiales de 2 a 7 , solitarios y agregados de 3 , moderadamente pocos de $5(3-10) / \mathrm{mm}^{2}$ y de diámetro tangencial mediano de 100 (50-130) $\mu \mathrm{m}$. Los elementos de vaso son muy largos de 1272 (869-1896) $\mu \mathrm{m}$, sus paredes muestran puntuaciones areoladas alternas y opuestas y placa perforada simple. La mayoría presenta gomas.

El parénquima es difuso en agregados, vasicéntrico, aliforme y aliforme confluente. $\mathrm{Al}$ gunas células con cristales romboidales.

Los rayos son biseriados 2 (1-3), heterogéneos, muy numerosos de $10(8-15) / \mathrm{mm}$, muy bajos de 691 (316-1185) $\mu \mathrm{m}$ y moderadamente finos de 35 (19-59) $\mu \mathrm{m}$. La mayoría presenta cristales romboidales.

Las fibras son de tipo libriforme, moderadamente largas de 1710 (1320-2220) $\mu \mathrm{m}$, de diámetro fino de 7 (6-9) $\mu \mathrm{m}$ y pared gruesa de $3(1-5) \mu \mathrm{m}$.

\section{Aspidosperma megalocarpon}

Familia: Apocinaceae

Nombres comunes: pelmax, peech ma' ax, peech nox, peel ma' pelmax (Sosa et al.1985, Pennington \& Sarukhán 1998).

Distribución en México: Se encuentra en la vertiente del Golfo, desde el centro de Veracruz, al norte de la Sierra de Naolinco, hasta la Península de Yucatán y en la vertiente del Pacífico de Guerrero a Oaxaca (Pennington \& Sarukhán 1998).

Características anatómicas de la madera:

a. Estéticas. La madera presenta diferencia de color entre albura y duramen, la albura es castaño rojizo (5YR 5/3) y el duramen es rojizo (5YR 4/3), no tiene olor ni sabor característicos, el brillo es bajo, de veteado suave, textura fina e hilo entrecruzado. Las zonas de crecimiento están marcadas por parénquima axial.

b. Macroscópicas y microscópicas. (Figs. 2. g-i). Todos los elementos constitutivos son visibles con lupa (6X). Los poros son de distribución difusa, la mayoría solitarios, muy numerosos de 48 (30-63)/mm $\mathrm{mm}^{2}$ y de diámetro tangencial moderadamente pequeño de 49 (30-80) $\mu \mathrm{m}$. Los elementos de vaso son medianos de 417 (284-632) $\mu \mathrm{m}$, sus paredes muestran puntuaciones areoladas alternas y placa perforada simple. La mayoría presenta gomas.

El parénquima es difuso en agregados, unilateral, vasicéntrico y en bandas confluentes.

Los rayos son uniseriados y multiseriados 3 (2-4), heterogéneos, muy numerosos de 12 (6-15)/ mm, extremadamente bajos de 200 (150-300) $\mu \mathrm{m}$ y moderadamente finos de 31 (15-49) $\mu \mathrm{m}$.

Las fibras son de tipo libriforme, medianas 'de 1170 (810-1570) $\mu \mathrm{m}$, de diámetro fino de 20 (10-21) $\mu \mathrm{m}$ y pared delgada de 6 (4-8) $\mu \mathrm{m}$. 

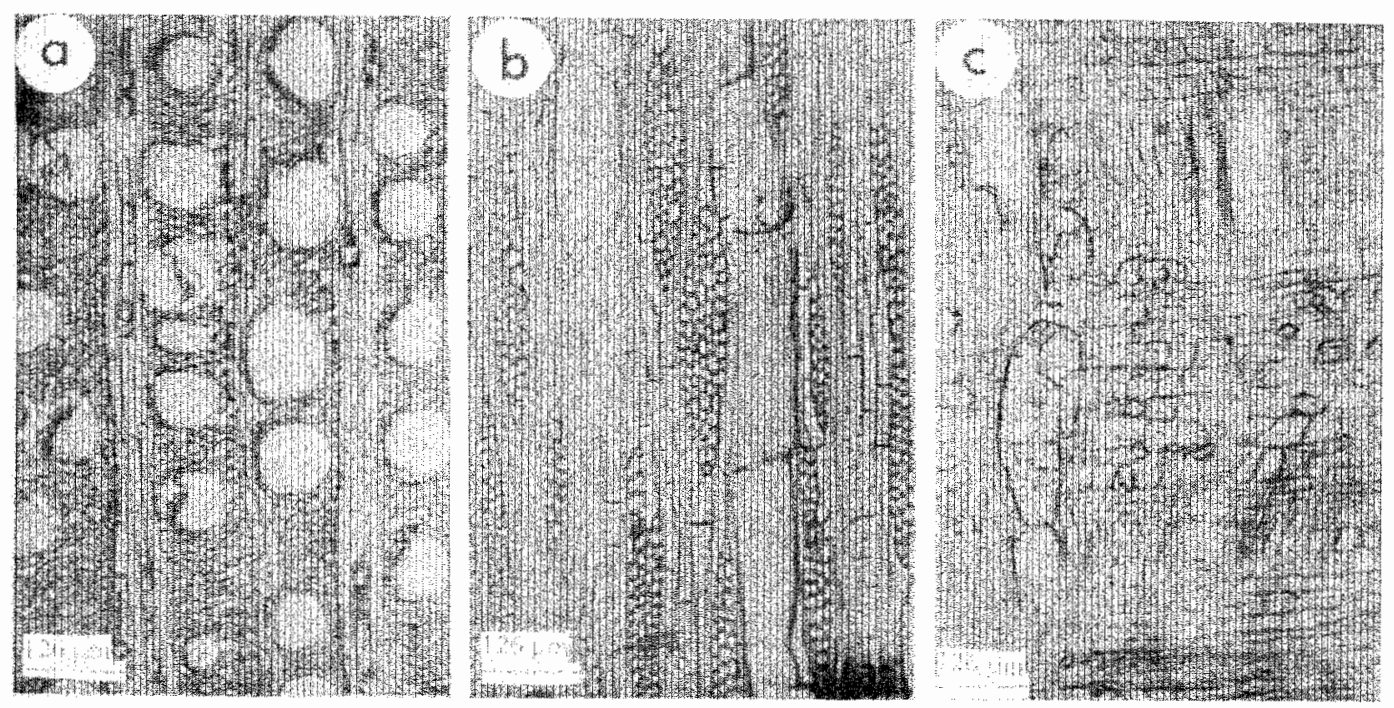

Fig. 3. Corte transversal (izquierda), corte tangencial (centro), corte radial (derecha). Figs. a-c. Ehretia tinifolia cupanioides.

Fig. 3. Transverse section (left), tangential section (centre), radial section (right). Figs. a-c. Ehretia tinifolia cupanioides.

\section{Ehretia tinifolia}

Familia: Boraginaceae

Nombres comunes: bek, beek, roble (Sosa et al. 1985).

Distribución en México: Yucatán, Campeche y Quintana Roo (Standley \& Steyermark 1949, Sosa et al. 1985).

Características anatómicas de la madera:

a. Estéticas. La madera no presenta diferencia de color entre albura y duramen, es castaño amarilla (10 YR 7/4), no tiene olor ni sabor característicos, su brillo es bajo, de veteado suave, textura fina e hilo entrecruzado. Las zonas de crecimiento están marcadas con fibras.

b. Macroscópicas y microscópicas. (Fig. 3. a-c). Todos los elementos constitutivos son visibles con lupa $(6 \mathrm{X})$. Los poros son de distribución difusa, exclusivamente solitarios, moderadamente numerosos de $16(13-18) / \mathrm{mm}^{2}$ y de diámetro tangencial moderadamente pequeño de 96 (79-110) $\mu \mathrm{m}$. Los elementos de vaso son extremadamente cortos de 72 (65-88) $\mu \mathrm{m}$, sus paredes muestran puntuaciones areoladas alternas y placa perforada simple. La mayoría presenta gomas.
El parénquima es apotraqueal en bandas de una hilera y paratraqueal escaso. Algunas células presentan sílice.

Los rayos son multiseriados de 3 (2-5) series, heterogéneos, muy numerosos de 7 (59)/mm, extremadamente bajos de 550 (440670) $\mu \mathrm{m}$ y medianos de 64 (48-80) $\mu \mathrm{m}$ La mayoría presenta cristales romboidales.

Las fibras son de tipo libriforme, extremadamente cortas de 280 (250-312) $\mu \mathrm{m}$, de diámetro ancho de 40 (32-48) $\mu \mathrm{m}$ y pared gruesa de 18 (15-20) $\mu \mathrm{m}$.

\section{DISCUSIÓN}

Las maderas estudiadas presentan características anatómicas que les confieren cualidades para ser usadas en diversas formas; en cuanto a las características estéticas presentan colores y veteados muy atractivos resaltando sobre todo sus contrastes entre albura y duramen, a excepción de $E$. tinifolia que es de color castaño amarillento y de $B$. cupanioides que es de color castaño muy pálido. E. tinifolia tiene veteado 
suave, textura fina y el hilo entrecruzado, que le proporciona resistencia mecánica a diferencia de $E$. latifolia que tiene hilo recto y textura mediana que le favorecen sea usada como madera tallada y entintada para artículos artesanales Guridi (1980); la presencia de gomas y sílice explica entre otras cosas que localmente se haya caracterizado como dura y muy durable así como el uso tradicional que tiene en la elaboración de cachas para rifles, mangos de herramientas tablas y aplicable a carpintería en general, otro uso conocido en el estado de Yucatán es como leña, Terán y Rasmussen (1994). La madera de B. cupanioides, es de veteado suave, textura fina e hilo recto por lo que es resistente al impacto, tiene gomas y cristales por lo que es considerada dura y es usada localmente en tablas para hacer muebles, sobretodo sillas se puede sugerir para la manufactura de pisos, escaleras, y artículos deportivos. C. cozumelensis y C. spicata son maderas de colores muy atractivos, rosa a castaño rojizos y gris rojizo a castaño pálido respectivamente, hilo entrecruzado, presencia de gomas en vasos, rayos y fibras así como cristales en el parénquima axial: por lo que responden bien al deterioro, lo que favorece que localmente son consideradas como muy durables por lo que son muy usadas en construcción rural de casas habitación, particularmente en los techos y para mangos de herramientas, uso que también tiene $C$. barbadensis de Chiapas, Andrade (1976) y de Quintana Roo, De la Paz Pérez et al. (1980); ambas presentan rayos uniseriados, numerosos, muy finos y extremadamente bajos, características que favorecen la resistencia al impacto y a la estabilidad dimensional, por lo que también son usadas para la elaboración de durmientes, sin embargo se pueden ampliar sus usos para pisos, parquet, adoquín, lambrin, para manufactura de muebles, puertas, marcos para ventanas, y decoración, , artículos torneados y decoración de interiores. El hilo entrecruzado aumenta las propiedades de resistencia mecánica por lo que son adecuadas para usos como pisos, construcción pesada, de yates y sobre todo en la decoración, lo que coincide con los usos recomen- dados por De la Paz Pérez et al. (1980). G. lucida, es una madera que se puede considerar como durable, presenta hilo entrecruzado que le confiere resistencia mecánica, las gomas en vasos y fibras, los cristales, y los diámetros de los vasos y de las fibras que son muy pequeños y muy finos respectivamente son caracteres que favorecen su durabilidad y su resistencia al agua, por lo que es usada en construcción rural como postes de palapas, en travesaños y en puntales de casas habitación; los rayos son muy numerosos, extremadamente bajos y muy finos, esto permite que sea considerada localmente como suave para cortar y es usada para hacer muebles; por estas características se puede sugerir para otro tipo de construcciones pesadas, como puentes, armazón de embarcaciones, pisos, escaleras, puentes, esculturas, usos que también son sugeridos por Rogel (1982). C. winterana, por las tonalidades de color es atractiva para la manufactura de muebles. presenta hilo entrecruzado que le proporciona resistencia mecánica, la mayoría de los vasos con gomas; se presentan cristales en el parénquima axial y en los rayos los cuales son muy numerosos, muy bajos y moderadamente finos, esto influye en las contracciones que sufre la madera y permite entender que localmente sea sugerida para durmientes, además las paredes gruesas de las fibras le confieren el peso y la dureza necesarias para usos de construcción pesada. En Puerto Morelos es usada para la construcción de palapas y tablas, es considerada como madera dura pesada y muy durable, características que coinciden con las de la madera de la misma especie de Cuba donde se puede emplear en postes de cerca, carbón, postes eléctricos y objetos torneados, Fors (1975). A. megalocarpon presenta hilo entrecruzado, vasos muy numerosos de diámetros pequeños y la mayoría con gomas, los rayos muy numerosos y extremadamente bajos y finos, estos caracteres favorecen que la madera sea considerada como muy dura, muy pesada y muy durable, razones que justifican que localmente sea muy usada para la construcción de casa rurales, en tablas y en durmientes; sin embargo las tonalidades de 
color castaño rojizos que tiene la hacen muy atractiva para ser usada en la manufactura de muebles, decoración, en pisos, parquet, lambrin, por su peso y dureza se recomienda para construcciones pesadas, para embarcaciones, esto coincide con los usos sugeridos para muestras de esta especie procedentes de Chiapas, Andrade (1976), De la Paz Pérez y Corral (1980) y de Campeche estudiadas por Huerta y Becerra (1982), que son usadas para chapa, fabricación de embarcaciones, pisos, artículos para atletismo y artículos deportivos, madera aserrada, durmientes y construcción pesada, las que se sugieren para ser usadas como madera terciada, armazones de canoas, en acabados, decoración de interiores, para fabricar muebles en ebanistería, para empaques de lujo y para elaborar mangos para herramientas. También se han realizado algunos ensayos tecnológicos en muestras procedentes de $\mathrm{Gu}$ yana y Guatemala que la clasifican como madera moderadamente resistente al ataque de hongos e insectos, fácil de trabajar con máquinas y herramientas de carpintería (Echenique-Manrique 1970) y Andrade (1976). Los resultados de los ensayos tecnológicos hechos por Herrera et al. (1982) la clasifican como no adecuada para elaborar zapatas de frenos del METRO.

\section{AGRADECIMIENTOS}

Las autoras agradecen al Equipo Técnico del Jardín Botánico del Centro de Investigaciones de Quintana Roo en Puerto Morelos, especialmente a Odilón Sánchez y David Garrido; a Alfonso Argüelles y a los Comisarios Ejidales de Noh-Bec y Tres Garantías, Luciano Pacheco Dorantes y Mateo Poot respectivamente, por las facilidades brindadas en el trabajo de colecta de las especies, así como al Departamento de Productos Forestales y Conservación de Bosques del Instituto de Ecología A. C., por el procesado de la madera, a Ingrid Olmsted y Rafael Durán por la determinación botánica de las especies. A Carmen de la Paz Pérez y Blanca Pérez-García por la revisión y valiosas sugerencias al manuscrito.

\section{RESUMEN}

Se describe la anatomía de la madera de Coccoloba cozumelensis, Coccoloba spicata, Gymnanthes lucida, Blomia cupanioides, Canella winterana, Aspidosperma megalocarpon y Ehretia tinifolia. La madera provino de árboles recolectados en la selva mediana subperennifolia en tres municipios del estado de Quintana Roo, México. Las especies son importantes porque tradicionalmente los campesinos de las diferentes localidades les han dado usos diversos a la madera en manufactura de muebles, herramientas, en construcción de casas habitación, en postes, cercas, tablones, durmientes y leña. Se hizo la descripción de la anatomía microscópica en preparaciones fijas de los tres cortes típicos de la madera y en material disociado; la macroscópica en tablillas de xiloteca. Las especies presentan porosidad difusa, puntuaciones areoladas alternas y placas simples, rayos numerosos, pequeños y finos; las fibras libriformes, la mayoría cortas y de diámetro fino; el material ergástico presente en forma de gomas, cristales de carbonato de calcio y sílice. La estructura anatómica de la madera les confieren cualidades estéticas, de peso, dureza, resistencia mecánica y al deterioro que responden a los usos tradicionales que en forma empírica les han dado los campesinos mayas y las hacen versátiles para destinarlas a diversos usos.

\section{REFERENCIAS}

Andrade. D.P. 1976. Aspectos ecológicos y usos de veintidós especies maderables de la Zona Lacandona de Chiapas. Tesis de Licenciatura. Universidad Nacional Autónoma de México. México, D.F.

Anónimo. 1937. Standar terms of size lenghts of vessel members and wood fibers. Trop. Woods 51:21.

Anónimo. 1939. Standar of size for vessel diameter and ray width. Trop. Woods 59: 51-52.

Anónimo. 1954. Munsell soil color charts. Munsell. Baltimore. Maryland. 17 p.

Anónimo. 1989. IAWA list of microscopic features for hardwood identification. IAWA Bulletin n.s. 10: 219-332.

Chattaway, M. 1932. Proposed standards for numerical value used in describing woods. Trop. Woods. 29: 20-28.

De Dalla Torre, C. \& H. Harms. 1963. Genera siphonogamarum ad systema Englerianum conscrypta. Akademischen Druck-Uberlagsanstalt .Graz. 637 p.

De la Paz Pérez-O. C.,T.F. Carmona \& A. Rogel. 1980. Estudio anatómico de la madera de 43 especies tropicales. Bol. Téc. Inst. Nac. Invest. For. 63. México, D.F. 276 p. 
De la Paz Pérez-O. C., \& G. Corral. 1980. Estudio anatómico de la madera de once especies de Angiospermas. Bol. Téc. Inst. Nac. Invest. For. 64. México, D.F. 79 p.

Echenique-Manrique, R. 1970. Descripción características y usos de 25 maderas tropicales mexicanas. Serie Maderas de México. Cámara Nacional de la Industria de la Construcción. México, D.F. 237 p.

Escalante, S. 1986. La Flora del Jardín Botánico del Centro de Investigaciones de Quintana Roo. A.C. Tesis de Licenciatura.Universidad Veracruzana. Xalapa, Ver. México.

Fors, A.J. 1975. Maderas Cubanas. Instituto Cubano del Libro y Facultad de Ciencias Agropecuarias. Universidad de la Habana. La Habana. 171 p.

Guridi, L. 1980. La madera en las artesanías del Estado de Michoacán. Bol. Div. Inst. Nac. Invest. For 50. México, D.F. 129 p.

Herrera, R. J., E. Barretero \& A. Herrera. 1982. Zapatas de maderas mexicanas para el sistema de frenos del "Metro". Bol. Tec. Inst. Nac. Invest. For. 76. México D.F. 59 p.

Huerta, J. \& J. Becerra. 1982. Anatomía macroscópica y algunas características físicas de diecisiete maderas tropicales mexicanas. Bol. Div. Inst. Nac. Invest. For. 46. México, D.F. 61 p.

Index Kewensis. 1997. Kew Royal Botanical Garden. Richmond.

Kribs, D.A. 1968. Commercial foreing woods on the american market. Dover; Nueva York. 241 p.

Martínez, M. 1979. Catálogo de nombres vulgares y científicos de plantas mexicanas. Fondo de Cultura Económica. México $1220 \mathrm{p}$.

Miranda, F. 1953. Plantas de Chiapas nuevas para la Flora Mexicana. Ann. Inst.Biól. Méx. 24 (1): 63-96.

Pennington, D. \& J. Sarukhán. 1998. Árboles Tropicales de México. Universidad Nacional Autónoma de México. Fondo de Cultura Económica. México, D.F. 521 p.
Ramos, C. \& V. Díaz. 1981. Instrucciones para colectar muestras de madera para estudios tecnológicos. Bol Div. Inst. Nac. Invest. For. 54. México, D.F. 15 p.

Rebollar, S., C. de la Paz Pérez-O. \& A. Quintanar. 1987. Maderas de la Península de Yucatán. México. Biótica 12: 159-179.

Rebollar, S., C. de la Paz-Pérez-O. \& A. Quintanar. 1993. Anatomía de la madera de cinco especies de Quintana Roo, México. Bol. Soc. Bot. Méx. 53: 103-124.

Rogel, M. 1982. Características anatómicas de la madera de siete especies tropicales. Bol. Téc. Inst. Nac. Invest. For. 86. México, D.F. 55 p.

Sosa, V., J.S. Flores, V. Rico-Gray, R. Lira \& J.J. Ortíz. 1985. Lista florística y sinonimia Maya 225 p. In: Etnoflora Yucatanense. Instituto Nacional de Investigaciones Sobre Recursos Bióticos. Fasc. 1. Xalapa, Veracruz, México.

Standley, P. C. \& J. A. Steyermark. 1946. Polygonaceae. 24: 112. In: Flora de Guatemala Fieldiana Bot. Field Mus. Nat. Hist. Part. IV. Chicago.

Standley, P.C. \& J. A. Steyermark. 1946a. Polygonaceae. 24: 117. In: Flora de Guatemala. Fieldiana Bot. Field Mus. Nat. Hist. Part. IV. Chicago.

Standley, P.C. \& J.A. Steyermark. 1949. Euphorbiaceae. 24: 120. In: Flora de Guatemala. Fieldiana Bot. Field Mus. Nat. Hist. Part. VI. Chicago.

Terán, S. \& Ch. Rasmussen. 1994. La Milpa de los Mayas. DANIDA. Gobierno del estado de Yucatán. Yucatán México. $345 \mathrm{p}$.

Tortorelli, L. 1956. Maderas y bosques argentinos. ACME. Buenos Aires, $910 \mathrm{p}$.

Villers, L., R.M. López-Franco \& A. Barrera. 1981. La unidad habitación tradicional campesina y el manejo de los recursos bióticos en el área maya yucatanense. Biótica 6(3): 293-323. 